\title{
L'ALALC, le marché commun de l'Amérique centrale et les intégrations européennes en droit comparé.
}

\author{
Rudolf Moser \\ Catedrático de Direito Internacional Privadc \\ na Universidade de Saint-Gall (Suiça) e na \\ Faculdade de Ciências Jurídicas do Rio de \\ Janeiro (Brasil).
}

I.

L'Alalc possède la personnalité juridique; L'art. $46 \mathrm{du}$ Traité de Montevideo de $1960^{1}$ le mentionne expressément en disant:

"La Asociación Latinoamericana de Libre Comercio gozará de completa personalidad juridica."

En poursuivant que l'Alalc est habilitée à conclure des traités, l'art. 46 la considère comme personne morale du droit international. Ce fait juridique devrait être confirmé par la conclusion de conventions ou par la reconnaissance formelle de l'Alalc en tant que membre de la communauté des nations par d'autres Etats. En effet, l'Alalc a conclu avec l'Uruguay un accord concernant les privilèges et les immunités de ses organes et du Secrétariat de Montevideo, comme prévu dans l'art. 47, al. 3, du Traité. Ce traité est analogue à celui que a été conclu entre la Suisse et l'Association Européenne de Libre Echange au sujet de son siège

1. Tratado que establece una zona de libre comercio e instituye la Asociación Latinoamericana de Libre Comercio, du 18 février 1960. 
de Genève, selon la Convention de Stockholm, art. 35, chiffre 2; l'art. 35 , ch. 1, parle de la capacité juridique de l'AEIEE'.

Quant à la Communauté Economique Européenne, l'art. 211 du Traité de Rome ${ }^{3}$ est ainsi conçu:

"Dans chacun des Etats membres, la Communauté possède la capacité juridique la plus large reconnue aux personnes morales par les législations nationales..."

Selon l'art. 228, la C.E.E. peut conclure des accords avec d'autres Etats ou des organisations internationales.

Il s'agit donc dans les trois cas d'un sujet du droit international. Ce qui n'est pas le cas pour l'Union de l'Amérique centrale. Le Traité de Manágua, du 13 décembre $1960,{ }^{4}$ ne parle nulle part dé personnalité juridique de l'organisation en tant que telle, tout en la reconnaissant expressément à son Secrétariat dans l'art. XXIII. Ce Secrétariat, "Secretaría permanente del Tratado General de Integración Económica Centroamericana" - appelé SIECA - a son siège à Guatemala City. C'est, en quelque sorte, la managing company pour l'intégration de l'Amérique centrale dépourvue de personnalité juridique. Cette organisation elle-même n'est ainsi, juridiquement parlant, qu'un simple traité de droit international. Conséquemment, à l'occasion du Seminario sur l'aspectes juridiques et institutionnales de la integration "centroamericana", organisé par l'Instituto Interamericano de Estudios Jurídicos Internacionales, tenu à Miami en août $1964,5^{5}$ on a étudié de quelle façon l'Union Economique de l'Amérique Centrale pourrait obtenir la

2. Convention instituant l'Association Européenne de Libre-Echange, du 4 janvier 1960 - "Convention de Stockolm".

3. Traité instituant la Communauté Economique Européenne, du 25 mars 1957.

4. Tratado General de Integración Económica Controamericana.

5. Cf. Informe de la Secretaría general/Report of the General Secretariat ch. 12. 
personnalité juridique du droit des gens et être ainsi habilitée à conclure des traités avec les pays tiers, les organisations internationales et les institutions privées.

\section{II.}

Il se pose la question de la supranationalité des quatre intégrations. Quel est le critère de la supranationalité? On peut reconnaître tel critère ${ }^{6}$ dans le fait qu'une autorité constituée par un traité promulgue, dans le cadre des compétences qui lui sont attribuées par ce traité, des normes matérielles ou de conflit qui ont force de loi dans le territoire de la communauté. Ce sera alors du droit communautaire, créé par l'autorité commune douée de pouvoir législatif ("autoridade supra-nacional ordenadora jurídica").

C'est, selon l'avis de la plupart auteurs, ce qui vaut pour le Traité de Rome; en effet, le Conseil des Ministres et la Commission jouissent, suivant les arts. 145s., 155s., d'un tel pouvoir législatif qui est certes limité à certains égards, mais qui est en principe indépendant des Etats membres. Ce dernier point concerne en particulier les décisions majoritaires du Conseil où les Etats membres peuvent être mis en minorité — possibilité actuellement renvoyée à plus tard par le veto de M. De Gaulle, quant aux matières importantes. Que néanmoins la Commission, avant tout, peut être considérée comme organe supranational, résulte du fait que, conformément à l'art. 157, ch. 2, elle "exerce ses fonctions en pleine indépendance, dans l'intérêt général de la Communauté."

Selon l'art. 32, ch. 5, et autres du Traité de Stockholm, le Conseil de l'AELE peut également prendre des décisions majoritaires. mais il n'a pas de fonctions législatifes pro-

6. RUdolf Moser, Direito internacional privado, Unificação do direito e Comunidade Econômica Européia in "Revista da Faculdade de Direito da Universidade de São Paulo", vol. 58, 1963, p. 93s., 98. 
prement dites. Par consèquent, d'après le critère établi plus haut, l'AeLE ne jouit pas de la supranationalité.

Il en va de même en ce qui concerne les intégrations latino-américaines: le Traité de Manágua prévoit comme organes un Conseil Economique, un Conseil Exécutif et un Secrétariat, voir les arts. XX s. Le Conseil Economique doit exécuter les décisions du Comité de l'Amérique Centrale pour la Coopération Economique. Le Conseil Exécutif est subordonné au Conseil Economique, et le Secrétariat, quoique pourvu de personnalité morale, est organe exécutif. Les Conseils Economique et Exécutif peuvent prendre des décisions majoritaires, mais il s'agit de simples "mesures" concernant la politique économique, l'intégration, la coordination et non de législation à proprement parler.

Il n'en est pas de même dans l'Alalc: il y a là des "normas comunitarias", donc de droit communautaire ${ }^{7}$ qui devrait être promulgué par la "Conferencia". Le Comité Permanent et le Secrétariat sont des organes exécutifs, et le Conseil des Ministres créé en 1966 est subordonné à la Conférence, cf. arts. 33 s. Selon l'art. 38, la Conférence prend ses décisions à la majorité des deux tiers pendant les deux premières années, mais sous réserve du droit de veto des autres membres ("siempre que no haya veto negativo"). 8 Après ces deux ans, la Conférence doit fixer le mode de vote pour l'avenir (art. 38, al. 2), ce qui a fait l'objet de la Résolution 68 (III) de la Conférence prise le 4 octobre 1963. Il a été décidé de conserver le mode actuel mais de supprimer successivement le droit de veto en ajoutant au besoin de nouveaux cas de vote non soumis au droit de veto à ceux existant déjà précédemment. ${ }^{9}$

7 Cf. Seminario sobre aspectos jurídicos e institucionales de la ALALC, organisé par l'Instituto Interamericano de Estudios Jurídicos Internacionales déjà cité, de Montevideo, en octobre 1965, Raport du Secrétariat Général, ch. 19.

8. L'abstention est donc considérée comme agrément tacite.

9. Art. 38, lettres a-c: budget, élections, date et lieu des séances. 
Quand on considère la liste des compétences de la Conférence, il semble cependant douteux que l'Alalc puisse promulguer, avec ou sans veto, du droit communautaire, comme l'affirment les "pregressistes" parmi les critiques juridiques de l'Alalc. L'art. 34 prévoit: a) mesures visant à l'exécution du Traité et à son application, b) négociations entre les partenaires, c) budget, d) règlement, e) nomination du président et du vice-président, f) nomination du secrétaire. Ce sont donc uniquement des compétences exécutives ou administratives. Seule la lettre g), selon laquelle la Conférence doit "entender en los demás asuntos de interés comun", pourrait être considérée comme la base étroite d'une compétence législative de la Conférence de l'Alalc. Il est donc permis d'hésiter avant d'affirmer sa supranationalité qu'on peut toutefois admettre en faisant preuve de bonne volonté.

III.

La vue d'ensemble esquissée sur les organes des quatre intégrations doit être complétée par l'observation que la C.E.E. possède un parlement, le Parlement Européen auquel n'incombent toutefois que des compétences consultative et de contrôle, cf. art. 137; ensuite une Cour de Justice qui a à juger des différends concernant la Communauté et des questions d'interprétation. Ni l'Aele ni les intégrations latinoaméricaines ne disposent d'un tel organe judiciaire; toutefois l'art. XXVI du Traité de Manágua prévoit la formation d'un Tribunal d'Arbitrage ad hoc. En plus d'autres mesures visant au renforcement institutionnel de l'organisation, le séminaire sur l'Alalc, de Montevideo, a discuté en 1965 de la création d'un organe de juridiction. ${ }^{10}$

10. Voir le Rapport, chiffre 10s., 12. 
IV.

La substance des Traités de Montevideo et de Manágua par rapport à la C.E.E. et à l'AELE paraît plus importante et plus intéressante que les questions plutôt théoriques qui viennent d'être examinées. Quant au contenu des traités on peut faire les remarques suivantes:

Le Traité de Montevideo prévoit la création d'une zone de libre échange dans un délai de douze ans, c'est-à-dire jusqu'en 1973, et les arts. 2 s. contiennent les dispositions nécessaires à ce but. La déclaration des présidents des Etats membres faite à Punta del Este en avril 1967 a postulé la création d'un marché commun à partir de 1970 et d'une union douanière jusqu'en 1985. L'AlaLc opérera alors sa fusion avec le marché de l'Amérique centrale.

Par contre, le Traité de Manágua a prévu dès le début un marché commun et une union douanière (art. 1), similaire à la C.E.E. (art. 9, al. 1), tandis que le Traité de l'Aele (art. 1 s.) et celui de l'Alalc ne prévoient qu'une zone de libre échange.

Fin ce qui concerne les impôts, les taxes et d'autres charges internes, l'AlaLc stipule l'égalité de traitement des importations avec la production indigène (art. 21), alors qu'en ce qui concerne les "privilèges" ("privilegios"), on ne prévoit que le régime du traitement le plus favorisé (art. 18). L'Union de l'Amérique centrale prescrit clairement l'égalité de traitement à accorder aux marchandises d'un Etat membre avec les produits indigènes, c'est-à-dire l'exemption de ces marchandises de toute mesure limitative ou autre (art. III, cf. également art. VII). Cette réglementation correspond avec celle de la C.E.E. (art. 95) et de l'Aele (art. 6).

La C.E.E. établit une défense de discrimination absolue pour des raisons de nationalité (art. 7), la libre circulation des travailleurs (arts. 48s.), le droit d'établissement 
des personnes physiques et morales ainsi que des sociétés commerciales (arts. 52s., 58), la liberté d'échange de services, de paiements et de capitaux (arts. 106s., 59s., 67s.).

Le Traité de Stockholm également le droit d'établissement (art. 16), celui de l'OCDE ${ }^{11}$ la libéralisation des services, des paiements et des capitaux.

Quant à ces matières, le Traité de Manágua ne parle que de libre échange monétaire (art. X), tandis que le Traité de Montevideo prévoit l'application de la clause de la nation la plus favorisée aux capitaux originaires de la zone (art. 20), ainsi que l'harmonisation des prescriptions concernant le trafic des capitaux et des prestations de service (art. 15).

L'établissement de conditions égales de concurrence est un objectif auquel, entre autres, les Traités de Rome et de Stockholm consacrent les dispositions concernant les cartels et les monopoles (arts. 85s., respectivement art. 15); on a émis à ce sujet quantité d'actes et de décisions exécutrices. Le Traité de Montevideo mentionne les conditions de concurrence à l'égard de l'harmonisation des règlements d'importation et d'exportation etc. (art. 15) et aussi dans l'art. 52, al. 1, en disant ici :

"Ninguna Parte Contratante podrá favorecer sus exportaciones mediante subsidios $u$ otras medidas que puedan perturbar las condiciones normales de competencia dentro de la Zona."

D’une façon analogue, le Traité de Manágua interdit d'établir des dispositions qui pourraient défavoriser les produits d'un Etat membre par rapport à ceux d'un autre, spécialement à ses propres produits (art. VII).

Ces interdictions de discrimination sont dirigées contre le législateur, c'est-à-dire contre l'administration publique et ne constituent point, par conséquent, une base appropriée pour un droit de cartels des zones d'intégration. Un tel

11. Accord concernant l'Organisation de Coopération et de Développement Economique, du 14 décembre 1960, art. 2, lettre d. 
droit n'existe pas jusqu'à présent, mais il y a des prescriptions nationales, par exemple la loi brésilienne $n^{\circ} 4137 \mathrm{du}$ 10 septembre 1962 concernant l'abus du pouvoir économique ("abuso do poder econômico"), appelée loi anti-trust. ${ }^{12}$

\section{V.}

Une dernière observation de droit comparé interesse particulièrement l'internationaliste: elle concerne le rapprochement des législations, spécialement en matière de droit commercial. C'est le problème posée par la diversité des réglementations nationales. Les intégrations européennes aussi bien que les intégrations latino-américaines peuvent amener, sinon à une unification, du moins à une harmonisation, à une coordination, à un "rapprochement" des droits nationaux et cela, parce que les néccessités économiques de l'intégration, c'est-à-dire la création de conditions de prix de revient analogues et l'élimination des distorsions qui pourraient survenir au marché commun l'exigent. Le degré du rapprochement des législations déjà effectué ou à effectuer varie considérablement; il dépend du degré de l'intégration déjà réalisée ou à réaliser ainsi que des bases juridiques de celle-ci.

La C.E.E. en tant que communauté supranationale poursuivant des fins économiques et politiques, fondée pour une période illimitée (art. 240) et ne pouvant être résiliée, a créé un droit de cartels uniforme supranational, ainsi que le projet d'une société européenne; elle est en train ou a l'intention de harmoniser, au moins partiellement, les lois nationales douanières et fiscales, les lois concernant le

12. Voir RUDOLF Moser, dans le Boletim informativo mensal da Câmara Suiça de Comércio e Indústria no Brasil, Rio de Janeiro, juilletaout 1963 , p. 1 s.. 
travail et l'établissement, la concurrence et les sociétés commerciales, le transfert de leur siège, leur fusion et leur reconnaissance internationale, ainsi que les lois sur la reconnaissance et l'exécution réciproques des décisions judiciaires et des sentences arbitrales. Elle se base sur les dispositions du Traité de Rome qui prévoient en termes généraux le rapprochement des législations nationales (art. 3 , lettre h, arts. 100 à 102) ou qui, en particulier, la chargent des tâches susmentionnées. En outre, l'art. 220 oblige les Etats membres à résoudre partie de ces problèmes par des accords mutuels.

Par contre, les textes de base touchant l'harmonisation des lois sont très succincts dans les trois autres unions: L'AELE - fondée pour une période limitée allant jusqu'au moment de sa fusion avec la C.E.E. et prévoyant un droit de retrait (art. 42) - ne parle pas expressis verbis de cette harmonisation, tout en la présumant pour certaines matières, notamment en ce qui concerne les pratiques commerciales restrictives (art. 15) et le droit d'établissement (art. 16). Quant au reste, ce sont les objectifs généraux qui entrent en ligne de compte (art. 2).

En ce qui concerne l'Alalc qui, comme la C.E.E., a une durée illimitée et qui, au contraire, accorde en tout temps le droit de retrait à ses membres (arts. 63/64), on peut considérer l'art. 15 du Traité de Montevideo comme le point de départ du rapprochement des législations (harmonisation des systèmes d'importation et d'exportation ainsi que des dispositions à l'égard des capitaux, des biens et des services)..$^{13}$ Ce sont les arts. 15 et 22 qui ont donné lieu à des efforts pour coordiner les lois fiscales et monétaires

13. Voir RUDOLF MOSER, Questōes de direito comercial latino-americano, Revista do Instituto de pesquísas e estudos jurídico-econômicosociais, Bauru (Estado de São Paulo), vol. 6, 1967, p. 133 s., 166, note 80 . 
dans le but de favoriser les investissements. ${ }^{14}$ Récemment, le professeur Manuel A. Vieira, conseiller du Secrétariat de l'Alalc, à Montevideo, ${ }^{15}$ affirme que l'art. 34, lettre g, autorisant la Conférence à disposer "en los demás asuntos de interés común", peut servir de base à tout rapprochement légal. ${ }^{16}$ Quoi qu'il en sait, les présidents ont décidé en avril 1967 à Punta del Este 1"“aproximación a las legislaciones nacionales en la medida requerida por el proceso de integración", et, le 2 septembre 1967, ${ }^{17}$ le Conseil des Ministres réuni à Asunción a chargé le Comité Exécutif d'établir une Jiste des priorités. Les sujets en question n'ont pas encree été nommés. En 1965, le Séminaire de Montevideo a recommandé avant tout l'harmonisation des législations concernant les matières suivantes: commerce extérieur, droit des transports, libre circulation, marques et patentes, droit des sociétés et droit des obligations, droit ouvrier, assurance sociale. Malheureusement rien n'a été réalisé jusqu'à ce jour, et on doit donc se baser encore aux prescriptions nationales.

Dans l'Union de l'Amérique centrale (dont la durée a été fixée à vingt ans suivis d'un droit de résiliation de cinq à cinq ans, art. XXXI), l'harmonisation des législations nationales est en train de s'effectuer, surtout dans le domaine de la législation ouvrière et fiscale, bien que dans

14. Cf. Segunda Reunión especial sobre aspectos jurídicos e institucionales de la inversión privada extranjera, Rio de Janeiro, 1965, Informe de la Secretaría general del Instituto de estudios jurídicos internacionales, Inter-American Institute of international legal studies, Washington, spécialement p. $18 \mathrm{~s}$.

15. La ALALC y el derecho privado internacional, Anuario del Instituto hispano-luso-americano de derecho internacional, vol. 2, p. 176 S., 185 .

16. Des commentaires juridiques aux intégrations latino-américaines font défaut. Il semble, en outre, que les relations de droit comparé entre les intégrations latino-américaines et européennes n'aient pas encore été examinées.

17 Resolución 193, cf. Boletín de la Integración, 1967, p. 481. 
le Traité de Manágua ne soient prévues que des compétences de caractère général. ${ }^{18}$

Le rapprochement des législations en tant que moyen juridique pour obtenir l'intégration économique est nécessaire, mais il est difficile à réaliser. L'exemple de l'Amérique centrale montre bien que l'intégration des économies et l'harmonisation des lois nationales sont plus facilement à atteindre dans le cadre d'un espace à dimensions restreintes que dans celui d'un continent. Pour cette raison, l'Alalc tâche de surmonter ses difficultés actuelles par des ententes subrégionales telles que le "Clube Andino".

18. Art. XX pour le Conseil Economique, art.XXII pour le Conseil Exécutif. 